\title{
A community-based aquatic exercise program to improve endurance and mobility in adults with mild to moderate intellectual disability
}

\author{
Renée M. Hakim', Michael D. Ross², ${ }^{2,}$ Wendy Runco ${ }^{3}$, Michael T. Kane ${ }^{3}$ \\ 'Department of Physical Therapy, University of Scranton, Scranton, PA, USA \\ 2Department of Physical Therapy, Daemen College, Amherst, NY, USA \\ ${ }^{3}$ Department of Physical Therapy, St. Joseph's Center, Scranton, PA, USA
}

The purpose of this study was to investigate the impact of a community-based aquatic exercise program on physical performance among adults with mild to moderate intellectual disability (ID). Twenty-two community-dwelling adults with mild to moderate ID volunteered to participate in this study. Participants completed an 8-week aquatic exercise program (2 days/wk, $1 \mathrm{hr} /$ session). Measures of physical performance, which were assessed prior to and following the completion of the aquatic exercise program, included the timed-up-and-go test, 6-min walk test, 30 -sec chair stand test, $10-\mathrm{m}$ timed walk test, hand grip strength, and the static plank test. When comparing participants' measures of physical performance prior to and following the 8-week aquatic exercise program, improvements were seen in all measures, but the change in scores for the 6-min walk test, 30 -sec chair stand test, and the static plank test achieved statistical significance $(P<0.05)$. An 8-week group aquatic exercise program for adults with ID may promote improvements in endurance and balance/mobility.

Keywords: Aquatic, Intellectual disability, Therapy

\section{INTRODUCTION}

Recent research has emphasized the importance of addressing the health-related needs of adults with intellectual disability (ID). With respect to defining this population, ID is characterized by significant limitations in intellectual functioning and adaptive behavior originating before the age of 18 (American Association of Intellectual and Developmental Disabilities, 2013). A study of physical fitness levels by Hilgenkamp et al. (2012b) in a large sample of adults with ID $(n=1,050)$ found that approximately two-thirds of the entire study population scored "below average" or "impaired." Even the youngest age groups (50-59 or 50-54 years) in this sample achieved similar or worse results than age groups 20 to 30 years older in the general population. Similarly, a literature review by Enkelaar et al. (2012) found consistent reports that balance and gait capacities are affected in persons with ID compared to their age-matched peers. These problems begin at a young age and remain present throughout the entire lifespan of persons with ID, with a relatively early occurrence of age-related decline. Low physical fitness levels in older adults with ID demonstrated that this group is prone to unnecessary premature loss of functioning and health problems. The authors stated that maintaining physical fitness in adults with ID should have priority in practice and policy.

In relation to promoting fitness, a recent systematic review by Bartlo and Klein (2011) found moderate to strong evidence that physical activity positively affected balance, muscle strength, and quality of life in individuals with ID. The authors also found that the research in this area needs to be translated into practice, specifically the development of physical activity programs that are adaptable to the needs of individuals with ID. Likewise, Howie et al. (2012) found that adults with ID have few physical activity
${ }^{*}$ Corresponding author: Michael D. Ross (D) http://orcid.org/0000-0003-1438-9814 Department of Physical Therapy, Daemen College, Amherst, NY 14226, USA Tel: +1-7076285950, Fax: +1-7168398537, E-mail: mross@daemen.edu Received: October 7, 2016 / Accepted: January 31, 2017
This is an Open Access article distributed under the terms of the Creative Commons Attribution Non-Commercial License (http://creativecommons.org/licenses/by-nc/4.0/) which permits unrestricted non-commercial use, distribution, and reproduction in any medium, provided the original work is properly cited. 
environmental resources and opportunities available to them, especially those not living in group homes. They emphasized that future interventions are needed to increase physical activity opportunities in this underserved population.

In order to meet the health needs of adults with ID, the availability of community-based fitness programs should be explored. These programs may include exercises mediated in a variety of settings. A pool-based aquatic exercise program is a potentially viable exercise medium for adults with ID. The advantages of aquatic exercise therapy for individuals with ID are multifaceted. The physical benefits include facilitated movement and decreased joint loading due to the pool's buoyancy, which also helped to mitigate the impact of poor balance and posture. Social stimulation and interaction are fostered by aquatic activities, and there is a motivating influence of the pool environment. Albert (2010) suggested that aquatic exercise may be perceived as more enjoyable by those with low exercise motivation. Accordingly, aquatic training programs would target both physical and motivational domains in individuals with ID. However, there is a lack of evidence on the impact of aquatic exercise on physical performance in this population. Therefore, the purpose of this study was to investigate the impact of a community-based aquatic exercise program on endurance, mobility and motivation for exercise in adults with mild to moderate ID.

\section{MATERIALS AND METHODS}

We conducted a pretest-postest cohort design on a convenience sample of 22 community-dwelling adults (9 women, 13 men) with mild to moderate ID (Table 1). The sample was recruited through key area provider agencies and all of the study participants were volunteers. Selection criteria included: age 21 or above, diagnosis of mild to moderate level ID, ability to comprehend and

Table 1. Baseline characteristics for adults with intellectual disabilities $(n=22)$ participating in an 8-week aquatic exercise program

\begin{tabular}{lc}
\hline Variable & Value \\
\hline Age $(\mathrm{yr})$ & $37.14 \pm 9.45$ \\
Gender & \\
Women & $9(41)$ \\
Men & $13(59)$ \\
Systolic blood pressure $(\mathrm{mmHg})$ & $120.76 \pm 10.46$ \\
Diastolic blood pressure $(\mathrm{mmHg})$ & $78.10 \pm 9.24$ \\
Heart rate (beats/min) & $88 \pm 18.01$ \\
Oxygen saturation level & $95.82 \pm 3.29$ \\
\hline
\end{tabular}

Values are presented as mean \pm stamdard deviation or number (\%). follow simple directions with the aid of the mentor and capable of independent ambulation with or without an assistive device. Individuals were excluded if they had any of the following: (a) pre-existing conditions (neuromuscular, cardiovascular, musculoskeletal) that would preclude safe participation in an aquatic exercise program, (b) pre-existing diagnoses of dementia, amnesic disorders, dissociative disorders, schizophrenia or other psychotic disorders, (c) open wounds, uncontrolled seizures, infectious diseases, incontinence, severe hydrophobia, medical instability, severely impaired temperature regulation, or cerebral hemorrhage less than 3 weeks prior to start of program, (d) known allergy to pool chemicals, or (e) uncontrolled behavior outbursts. These selection criteria were communicated during recruitment of participants. In addition, screening was also conducted in person at the initial testing session by one of the investigators to definitively determine if each individual was eligible to participate. This project was approved by the Institutional Review Boards at the University of Scranton (Scranton, PA, USA) for the protection of human subjects prior to implementation.

All testing was completed during 2-hr sessions (pretest and posttest) at St Joseph's Center (Scranton, PA, USA). Physical performance testing was conducted by graduate physical therapy (PT) student research assistants (RAs) under the direct supervision of a licensed PT. These RAs were trained on standardized procedures for the selected physical performance tests prior to the start of this study. All testing was carried out by a pair of RAs at each station; one to provide instructions and record data and the other to guard for safety. Participants rotated to each station in a random order until all testing was completed as described below.

\section{Tests and measures}

Each participant had resting heart rate and blood pressure measurements taken in sitting. Heart rate was measured using palpation of the radial pulse at the wrist for $15 \mathrm{sec}$. Blood pressure was recorded using a standard sphygmomanometer and stethoscope.

The 6-min walk test (6MWT) was used to measure cardiovascular endurance. The 6MWT is a well-established test of submaximal aerobic capacity/endurance that assesses distance walked over $6 \mathrm{~min}$. The test was performed at the fastest comfortable speed possible. Nasuti et al. (2013) modified the 6MWT for adults with ID to include familiarization, a 1:1 pacer, and encouragement every $15 \mathrm{sec}$. They found that the modified 6MWT was easy to administer and score and showed acceptable validity in relation to $\mathrm{VO}_{2}$ peak $(r=0.84, P<0.001)$ and excellent reliability (intraclass correlation coefficient $[$ ICC $]=0.98$ ) for assessing cardiovascular 
fitness in adults with ID. The distance from one trial was used for data analysis.

The 10-m timed walk (10MTW) was used to measure gait velocity. Each participant walked a distance of $10 \mathrm{~m}$ with the time measured for the central $6 \mathrm{~m}$ to allow for acceleration and deceleration at each end. Gait velocity was measured for three trials of self-selected, preferred walking pace. A stop watch was used to time walking that occurs in the marked area. Distance divided by time was calculated for each trial which indicates gait velocity $(\mathrm{m} /$ $\mathrm{min}$ ). Gait speed measures were found to be highly reliable, (ICC $=0.90$ and ICC $=0.91$ for comfortable and maximum speed, respectively; Bohannon, 1997). In addition, testing of gait speed was found to be feasible and reliable (ICC $=0.63-0.96$ ) in adults with mild to moderate ID (Enkelaar et al., 2013; Hilgenkamp et al., 2012a). The mean of three trials was used for data analysis.

The timed-up-and-go (TUG) was used to measure balance/mobility. The TUG measures the time required to stand, walk at a self-selected/usual pace for three meters, turn, walk back to the chair and sit down. The interrater $(I C C=0.99)$ and test-retest (ICC $=0.98)$ reliability have been established for the TUG (Podsiadlo and Richardson, 1991). Concurrent validity has been reported in relation to log-transformed scores on the Berg Balance Scale $(r=-0.81)$, gait speed $(r=-0.61)$ and the Barthel Index $(r=-0.78)$ (Podsiadlo and Richardson, 1991). In addition, this test was found to be feasible and reliable (ICC $=0.63-0.96$ ) for mobility and balance testing in adults with ID (Enkelaar et al., 2013; Hilgenkamp et al., 2012a). The TUG was performed 2 times, with the second trial time used for data analysis.

The 30-sec chair stand (30 SCS) is a physical performance test used to measure lower extremity strength, balance and mobility. The participant, with arms folded across the chest and feet shoulder-width apart, completed as many full stands as possible in 30 sec. Reliability has been established for test-retest interclass correlations for men $(r=0.84)$ and for women $(r=0.92)$. Criterion validity has been reported in relation to the maximum weight-adjusted leg-press performance for both men $(r=0.78)$ and women $(r=0.71)$ as an indicator of lower body strength (Jones et al., 1999). This test was found to be feasible and reliable (ICC $=0.63-$ 0.96) as part of a fitness testing battery in a sample of adults with ID (Hilgenkamp et al., 2012a). A single trial was performed with a standard, armless chair.

The static plank test was used as an assessment of core body strength. It required a participant to hold the body off the ground while supported by toes and forearms for $60 \mathrm{sec}$. Each participant was instructed to keep the elbows directly below the shoulders with forearms and fingers extended straight forward with the neck in neutral and the body extended in a straight line. The plank test was reported to have high test-retest reliability $(r=0.80)$ when used in young adults with ID to assess fitness (Barwick et al., 2012). If the participant was able to assume the basic plank position, a single trial for a maximum of $60 \mathrm{sec}$ was recorded for data analysis.

Each participant performed a dominant handgrip strength assessment using a Jamar hand grip dynamometer (Patterson Medical, Warrenville, IL, USA). Testing was performed in sitting, with shoulder and wrist in neutral and elbow at the 90 degree position. Three trials of maximal grip strength for the dominant hand were assessed with the mean recorded for data analysis.

Upon entry into the study, the primary care physician of each participant was contacted. A RA faxed a letter to each participant's doctor to provide notification that his/her patient had enrolled in this study. There was information provided to allow contact of the primary investigator with any questions or concerns. Attendance was taken at each session and follow-up contacts (phone and/or email) were made with those not in attendance. Detailed adherence records were recorded along with reasons for nonattendance.

\section{Intervention}

The intervention consisted of an 8-week aquatic exercise program conducted at St. Joseph's Center. The sessions were offered twice weekly ( $45 \mathrm{~min}$ to $1 \mathrm{hr}$ duration). This schedule was selected based on practicality and available resources. Elmahgoub et al. (2011) found that exercising 2 times each week was more feasible and practical for participants and had the same beneficial health effects as 3 times each week in a sample of sedentary adolescents with ID who were undergoing short-term training (10 weeks).

The aquatic therapy was supervised and led by a licensed PT with 1:1 guidance by PT students with each participant in the pool. These PT student "water buddies" were used to encourage participation in the aquatics program. A study by Temple and Stanish (2011) utilized a peer-guided model to provide reciprocal support during structured, individualized exercise sessions for youth with ID that were led by certified fitness trainers. Adherence to this community-based exercise program was high and participants showed a significant reduction in personal barriers. They also reported learning new skills and making new friends.

Our program was developed using a health behavior theory, social cognitive theory (SCT) (Bandura, 1986), as a framework. This theory addresses both the psychosocial dynamics influencing health behavior and methods of promoting behavioral change. The 
SCT was used successfully in a study by Elinder et al. (2010) for a health intervention that aimed to improve diet and physical activity in a group of adults with ID living in community residences. SCT suggests that an individual's behavior change can be directly related to observing others within the context of social interactions and experiences. Health behavior can be described in terms of this dynamic and reciprocal model in which behavior, personal factors, and environmental influences all interact. Based on SCT, the aquatic program may improve health behavior through changes in both personal (skills, preferences, self-efficacy) and social environmental factors. A licensed PT led all of the aquatic exercise sessions using SCT constructs. At the start of the program, the instructor defined the program expectations with respect to the schedule and activities. The outcome expectancies portrayed fitness as meaningful and functional for all participants. Reinforcements were given via praise and encouragement from "water buddies" and the instructor. Self-efficacy was targeted as participants gained task-specific confidence as they implemented the exercises and progressed through the program. The small group format helped participants to develop behavioral capabilities with observational learning as "water buddies" guided them through each exercise and provided vicarious learning opportunities for them to reproduce the same actions. Verbal persuasion was used to encourage each activity, as well as continued participation/adherence to the 8-week program. Please refer to Table 2 for aquatic program details.

\section{Data analysis}

To evaluate the effects of the aquatic exercise program on physical performance, paired $t$-tests were used to compare participants' measures of physical performance prior to and following the aquatic exercise program. The alpha value was 0.05 .

\section{RESULTS}

Subjects participated in $94 \%$ of the aquatic exercise training sessions (range, $87 \%$ to $100 \%$ ), suggesting a high adherence rate for the program. There were no adverse outcomes or events, such as falls, mechanical injuries or feeling unwell during aquatic exercise training sessions, suggesting that the aquatic exercise program was safe for participants. When comparing participants' measures of physical performance prior to and following the 8 -week aquatic exercise program, improvements were seen in all measures, but the change in scores for the 6-min walk test, 30-sec chair stand test, and the static plank test achieved statistical significance $(P<0.05)$ (Table 3).
Table 2. Overview of aquatic exercise program for community-dwelling adults with intellectual disabilities

\begin{tabular}{|c|c|}
\hline $\begin{array}{l}\text { General activity/body } \\
\text { region }\end{array}$ & Exercise \\
\hline Warm-up activities & $\begin{array}{l}\text { Lap walking × } 4 \text { laps each } \\
\text { Walking forward } \\
\text { Walking backward } \\
\text { Sidestepping } \\
\text { Lunging/long-stepping } \\
\text { Marching }\end{array}$ \\
\hline Upper body & $\begin{array}{l}\text { Bilateral upper extremity scaption } \\
\text { Flies (horizontal abduction/adduction) } \\
\text { Rows } \\
\text { Forward press (shoulder flexion/extension) } \\
\text { Lateral press (shoulder abduction/adduction) } \\
\text { Biceps/triceps exercises } \\
\text { "UBE" forward and backward × } 30 \text { sec each direction } \\
\text { Note: Were performed with or without resistance } \\
\text { paddles - } 2 \text { sets of } 10 \text { repetitions each }\end{array}$ \\
\hline Lower body & $\begin{array}{l}\text { Hip movements: flexion, extension, abduction, } \\
\text { adduction } \\
\text { Heel/toe lifts } \\
\text { Squats } \\
\text { Step-ups } \\
\text { Note: Were performed with and without ankle } \\
\text { weights - } 2 \text { sets of } 10 \text { repetitions each }\end{array}$ \\
\hline Trunk & $\begin{array}{l}\text { Rotation } \\
\text { Sidebending } \\
\text { Wall Push-ups } \\
\text { Note: Two sets of } 10 \text { repetitions each }\end{array}$ \\
\hline Cardiovascular activities & $\begin{array}{l}\text { Jog in place } \\
\text { Jumping jacks } \\
\text { Cross country skiing } \\
\text { Hopping: } \\
\text { Forward/backward } \\
\text { Left/right } \\
\text { Note: Thirty sec for each activity, progressing to } 2 \text { sets }\end{array}$ \\
\hline Optional activities & $\begin{array}{l}\text { Stationary swim kicks while prone on kickboard/ } \\
\text { noodle/pool wall } \\
\text { Cycling in noodle }\end{array}$ \\
\hline
\end{tabular}

UBE, upper body ergometer.

\section{DISCUSSION}

Our program targeted a group of underserved adults with ID in the community who no longer receive the support services that they qualified for during childhood and adolescence in accordance with the Individuals with Developmental Disabilities Education Act of 1990 (IDEA). IDEA provides all children with intellectual and developmental disabilities (IDD) the right to a free and appro- 
Table 3. Means and standard deviations for measures of physical performance at the time of the initial assessment and at the week 8 assessment for a sample of adults with intellectual disabilities $(\mathrm{n}=22)$

\begin{tabular}{lccc}
\hline Measure of physical performance & Initial assessment & Week 8 assessment & Difference \\
\hline 6-min walk test $(\mathrm{m})$ & $292.96 \pm 134.37$ & $319.50 \pm 152.54$ & $12.18 \pm 7.78$ \\
10-m timed walk test (sec) & $13.85 \pm 10.58$ & $18.08 \pm 15.23$ & 1.66 \\
Timed-up-and-go test (sec) & $20.14 \pm 16.17$ & $9.93 \pm 3.81$ & 2.06 \\
30-sec chair stand test (repetition) & $8.64 \pm 3.02$ & $17.21 \pm 23.41$ & $1.30^{*}$ \\
Static plank test (sec) & $11.23 \pm 17.17$ & $23.28 \pm 11.24$ & $5.98^{*}$ \\
Handgrip strength test (kg) & $22.30 \pm 11.05$ & 0.99 \\
\hline
\end{tabular}

Values are presented as mean \pm stamdard deviation.

Difference $=$ week 8 assessment-initial assessment.

${ }^{*} P<0.05$.

priate education and to any services deemed necessary to meet their needs. This provision of services is most comprehensive in the educational setting and ends after the 21st year. Among adults with ID over age 21 , the deinstitutionalization movement has dramatically increased the population residing outside of institutions in community-based settings, living either with family members or in supported-living, small group, residential settings (Mansell and Ericsson, 1996). With the rapid increase in need, expansion of community-based service delivery programs for adults with ID has not kept pace with demand. Shaw et al. (2011) found a need for mechanisms for community-dwelling adults with ID to maintain and increase social networks. Our aquatic exercise program addressed the need to improve the health and activity level of adults with ID age 21 and over while also providing a social opportunity.

Our sample of adults with ID improved across all measures of physical performance, with statistically significant gains noted for the 6MWT, 30 SCS, and the static plank test. These findings are consistent with a study of group aquatic exercise in children with IDD that found significant improvements in cardiorespiratory endurance (i.e., half-mile walk/run) and exercise capacity following a 14-week program (Fragala-Pinkham et al., 2008). In our study, the ability to walk greater distances, complete more chair stands and hold a plank position longer may be related to improved endurance, balance/mobility, and core body strength seen by participants following completion of the 8-week group aquatic exercise program. Significant changes were not seen for the 10MTW or the TUG, which were tests that were shorter in endurance compared 6MWT and 30 SCS. Given the nature of this training program in terms of potentially developing endurance (2 days/week, $1 \mathrm{hr} /$ session), it is not necessarily surprising that tests that required greater endurance (e.g., 6MWT, 30 SCS) were affected to a greater extent. Further research is necessary to determine the specific aspects of physical performance that are positively impacted through aquatic training for individuals with ID.

Our program utilized a theory of health behavior as a framework for the intervention. Based on application of SCT, the aquatic exercise program attempted to improve health behavior through changes in both personal (skills, preferences, self-efficacy) and social environmental factors (peer modeling, "water buddies"). SCT suggests that an individual's behavior change can be directly related to observing others within the context of social interactions and experiences. Our program provided support and social interaction to promote behavior change and subsequently led to a $94 \%$ group adherence/attendance rate. A systematic review by Hutzler and Korsensky (2010) found that improved physical fitness and elevated skill level gained during exercise and sport activities appear to serve as mediators for increased perceptions of self-efficacy and social competence and peer modeling appear to be important factors in maintaining adherence to exercise programs. Additional research is necessary to determine which theory of health behavior is the most appropriate to promote behavior change in adults with ID.

There are several limitations to this study. If the aquatic training group was compared to a control group, it may have given a more accurate account of the effects of aquatic training. A longer follow-up period would also have been informative to determine if there are any long-term effects of the aquatic training program on physical performance. Another limitation of this study was the small number of participants. In addition, this was a somewhat heterogenous sample of participants, in terms of factors such as age, intellectual ability, and functional capacity which may have potentially influenced the outcome. We also did not assess whether intelligence improved after aquatic exercise program. Future randomized controlled studies should be conducted with a larger sample size in order to confirm the results of our study.

An 8-week group aquatic exercise program for communi- 
ty-dwelling adults with ID may promote improvements in endurance and balance/mobility. The pool environment also provided a safe, motivational setting with an opportunity for social interaction. Future research should include a randomized, controlled clinical trial with a larger sample size to determine the most optimal training parameters for this target population.

\section{CONFLICT OF INTEREST}

No potential conflict of interest relevant to this article was reported.

\section{REFERENCES}

Albert PT. Alternative exercise may be attractive to more individuals. Home Health Care Manag Pract 2010;22:301-304.

American Association of Intellectual and Developmental Disabilities. Definition of intellectual disability [Internet]. Washington, DC: American Association of Intellectual and Developmental Disabilities; c2013 [cited 2016 Oct 6]. Available from: http://aaidd.org/intellectual-disability/definition\#.WljO_xszWcw.

Bandura A. Social foundations of thought and action: a social cognitive theory. Englewood Cliffs (NJ): Prentice Hall; 1986.

Bartlo P, Klein PJ. Physical activity benefits and needs in adults with intellectual disabilities: systematic review of the literature. Am J Intellect Dev Disabil 2011;116:220-232.

Barwick RB, Tillman MD, Stopka CB, Dipnarine K, Delisle A, Sayedul Huq M. Physical capacity and functional abilities improve in young adults with intellectual disabilities after functional training. J Strength Cond Res 2012;26:1638-1643.

Bohannon RW. Comfortable and maximum walking speed of adults aged 20-79 years: reference values and determinants. Age Ageing 1997;26: 15-19.

Elinder LS, Bergström H, Hagberg J, Wihlman U, Hagströmer M. Promoting a healthy diet and physical activity in adults with intellectual disabilities living in community residences: design and evaluation of a cluster-randomized intervention. BMC Public Health 2010;10:761.

Elmahgoub SS, Calders P, Lambers S, Stegen SM, Van Laethem C, Cambier DC. The effect of combined exercise training in adolescents who are overweight or obese with intellectual disability: the role of training frequency. J Strength Cond Res 2011;25:2274-2282.
Enkelaar L, Smulders E, van Schrojenstein Lantman-de Valk H, Geurts $\mathrm{AC}$, Weerdesteyn V. A review of balance and gait capacities in relation to falls in persons with intellectual disability. Res Dev Disabil 2012;33:291-306.

Enkelaar L, Smulders E, van Schrojenstein Lantman-de Valk H, Weerdesteyn V, Geurts AC. Clinical measures are feasible and sensitive to assess balance and gait capacities in older persons with mild to moderate Intellectual Disabilities. Res Dev Disabil 2013;34:276-285.

Fragala-Pinkham M, Haley SM, O’Neil ME. Group aquatic aerobic exercise for children with disabilities. Dev Med Child Neurol 2008;50:822827.

Hilgenkamp TI, van Wijck R, Evenhuis HM. Feasibility and reliability of physical fitness tests in older adults with intellectual disability: a pilot study. J Intellect Dev Disabil 2012a;37:158-162.

Hilgenkamp TI, van Wijck R, Evenhuis HM. Low physical fitness levels in older adults with ID: results of the HA-ID study. Res Dev Disabil 2012b;33:1048-1058.

Howie EK, Barnes TL, McDermott S, Mann JR, Clarkson J, Meriwether RA. Availability of physical activity resources in the environment for adults with intellectual disabilities. Disabil Health J 2012;5:41-48.

Hutzler Y, Korsensky O. Motivational correlates of physical activity in persons with an intellectual disability: a systematic literature review. J Intellect Disabil Res 2010;54:767-786.

Jones CJ, Rikli RE, Beam WC. A 30-s chair-stand test as a measure of lower body strength in community-residing older adults. Res Q Exerc Sport 1999;70:113-119.

Mansell J, Ericsson K, editors. Deinstitutionalization and community living: intellectual disability services in Britain, Scandinavia and the USA. London: Chapman and Hall 1996.

Nasuti G, Stuart-Hill L, Temple VA. The six-minute walk test for adults with intellectual disability: a study of validity and reliability. J Intellect Dev Disabil 2013;38:31-38.

Podsiadlo D, Richardson S. The timed "Up \& Go": a test of basic functional mobility for frail elderly persons. J Am Geriatr Soc 1991;39:142148.

Shaw K, Cartwright C, Craig J. The housing and support needs of people with an intellectual disability into older age. J Intellect Disabil Res 2011;55:895-903.

Temple VA, Stanish HI. The feasibility of using a peer-guided model to enhance participation in community-based physical activity for youth with intellectual disability. J Intellect Disabil 2011;15:209-217. 